\title{
Upper Limits on Sparticle Masses from $g-2$ and the Possibility for Discovery of Supersymmetry at Colliders and in Dark Matter Searches
}

\author{
Utpal Chattopadhyay \\ Department of Theoretical Physics, Tata Institute of Fundamental Research, Homi Bhabha Road, Mumbai 400005, India
}

Pran Nath*

Theoretical Physics Division, CERN, CH 1211, Geneva, Switzerland

(Received 14 February 2001; revised manuscript received 28 March 2001)

\begin{abstract}
We analyze the implications of the new physics effect seen in the $g-2$ Brookhaven measurement and show that if the effect arises from supersymmetry, then the sign of the Higgs mixing parameter $\mu$ is determined to be positive in the standard sign convention. Further, analyses within the minimal supergravity model show that the Brookhaven result leads to upper limits on the universal gaugino and scalar masses of $m_{1 / 2} \leq 800 \mathrm{GeV}$ and $m_{0} \leq 1.5 \mathrm{TeV}$ for $\tan \beta \leq 55$. Our analysis strongly suggests that supersymmetry via production of sparticles must be found at the CERN Large Hadron Collider. Further, $\operatorname{sgn}(\mu)$ positive is favorable for the discovery of supersymmetric cold dark matter.
\end{abstract}

\section{DOI: $10.1103 /$ PhysRevLett.86.5854}

The anomalous magnetic moment of the muon $a_{\mu}=$ $\left(g_{\mu}-2\right) / 2$ [where $g$ relates the magnetic moment $\vec{\mu}$ of a particle to its spin $\vec{S}$ by $\vec{\mu}=g(e / 2 m) \vec{S}]$ is one of the most accurately determined quantities in physics. Recently, the experiment E821 at the Brookhaven National Laboratory (BNL) has made a more precise determination of $a_{\mu}$ [1]. The new measurement is in good agreement with the previous determinations, but the combined error is now reduced by a factor of about 3 . The world average of the experimental results including the new measurements is given by $a_{\mu}^{\exp }=11659203(15) \times 10^{-10}$. The standard model prediction for $a_{\mu}$ including up to $\alpha^{5}$ QED corrections, $\alpha^{2}$ and $\alpha^{3}$ hadronic corrections, and up to two loop electroweak corrections gives [2] $a_{\mu}^{\mathrm{SM}}=11659159.6(6.7) \times 10^{-10}$ where essentially the entire error in the standard model prediction comes from the error in the hadronic corrections [3]. Remarkably the new Brookhaven measurement finds a $2.6 \sigma$ difference between the experiment and the standard model result [2] signaling the possible onset of new physics [1], i.e.,

$$
a_{\mu}^{\exp }-a_{\mu}^{\mathrm{SM}}=43(16) \times 10^{-10} .
$$

It has been known for some time that $a_{\mu}$ is sensitive to new physics such as supersymmetry (SUSY) [4-6]. Specifically, estimates of the correction in the well motivated supergravity unified (SUGRA) model showed in 1983-1984 that the supersymmetric correction to $a_{\mu}$ can be as large or larger [6] than the standard model electroweak correction $[2,7]$. The more recent analyses [8-10] support the previous conclusions [6] that the supersymmetric electroweak effects can be large. Further, it is known that large $C P$ effects can be consistent with the electron and the neutron electric dipole moment constraints [11] and analyses show that the $C P$ violations can generate large corrections to $a_{\mu}$ [12]. A variety of other effects such as arising from extra dimensions, anomalous $W$ couplings, etc. have also been examined (for a review, see Ref. [13]). In the following
PACS numbers: 13.40.Em, 12.60.Jv, 14.60.Ef, 95.35.+d

we analyze the implications of the new result from Brookhaven on sparticle masses. First, we show that the Brookhaven experiment determines the sign of the Higgs mixing parameter $\mu$. We then analyze the limits on the sparticle spectrum by using the constraint of Eq. (1), taking a $2 \sigma$ error corridor and attributing the entire difference between theory and experiment to supersymmetry, which gives $10.6 \times 10^{-10}<a_{\mu}^{\text {SUSY }}<76.2 \times 10^{-10}$, where $a_{\mu}^{\mathrm{SUSY}}=a_{\mu}^{\exp }-a_{\mu}^{\mathrm{SM}}$. For the purpose of definiteness we give an analysis of the constraint mostly within the framework of the SUGRA model and specifically in its minimal form (mSUGRA) [14]. However, for comparison we also discuss the results within the minimal anomaly mediated supersymmetry breaking (AMSB) scenario following the analysis of Ref. [10]. At low energy mSUGRA can be parametrized by $m_{0}, m_{1 / 2}, A_{0}, \tan \beta, \operatorname{sgn}(\mu)$, where $m_{0}$ is the universal scalar mass, $m_{1 / 2}$ is the universal gaugino mass, $A_{0}$ is the universal trilinear coupling at the grand unified theory scale, and $\tan \beta=\left\langle H_{2}\right\rangle /\left\langle H_{1}\right\rangle$, where $\left\langle H_{2}\right\rangle$ gives mass to the up quark and $\left\langle H_{1}\right\rangle$ gives mass to the down quark and the lepton, and $\mu$ is the Higgs mixing parameter which appears in the superpotential in the form $W^{(2)}=\mu H_{1} H_{2}$ (our sign convention on $\mu$ is that of Ref. [15]). The supersymmetric contributions $a_{\mu}^{\text {SUSY }}$ at the one loop level consist of the chargino-sneutrino exchange and of the neutralinosmuon exchange so that $a_{\mu}^{\mathrm{SUSY}}=a_{\mu}^{\tilde{\chi}^{ \pm}}+a_{\mu}^{\tilde{\chi}^{0}}$. However, typically it is the chargino-sneutrino exchange contribution $a_{\mu}^{\tilde{\chi}^{ \pm}}$that dominates.

It was noted in two previous papers several years ago [see Ref. [8] and Chattopadhyay and Nath $(\mathrm{CN})$ in Ref. [9]] that the sign of $a_{\mu}^{\mathrm{SUSY}}$ is correlated with the sign of $\mu$. It was shown by $\mathrm{CN}$ in Ref. [9] that this correlation arises because of the signature carried by the contribution of the light chargino exchange term in the chiral left and the chiral right interference term in the chargino exchange contribution. It was found that over most of the parameter space one has $a_{\mu}^{\mathrm{SUSY}}>0$ for $\mu>0$ and $a_{\mu}^{\mathrm{SUSY}}<0$ and 
for $\mu<0$, except when $\tan \beta$ is very close to 1 . Since the data from the BNL experiment indicate $a_{\mu}^{\mathrm{SUSY}}>0$ we conclude that

$$
\mu>0 \quad \text { (from BNL data [1]). }
$$

The fact that the sign of $\mu$ as determined by the BNL data turns out to be positive is of great consequence. It is known that the current experimental limits on the flavorchanging neutral-current process $b \rightarrow s \gamma$ eliminate a majority of the parameter space for $\mu<0$ [16] and consequently the neutralino-proton cross sections $\left(\sigma_{\chi^{0}-p}\right)$ for the direct detection of dark matter are significantly smaller. Current direct search experiments are sensitive to $\sigma_{\chi^{0}-p} \geq$ $1 \times 10^{-6} \mathrm{pb}$ and one expects future experiments to reach a sensitivity of $\sigma_{\chi^{0}-p} \geq 1 \times\left(10^{-9}-10^{-10}\right) \mathrm{pb}$ and this sensitivity can probe a majority of the parameter space of mSUGRA for $\mu>0$. However, this is not the case for $\mu<0$ [17]. Thus a positive $\mu$ sign given by Eq. (2) using the BNL data is very encouraging for the discovery of neutralino cold dark matter $[16,17]$.

We discuss now the other consequences of the BNL $g-2$ constraint. In Fig. 1 we plot the allowed region in the sneutrino and the light chargino plane for $\mu>0$ for values of $\tan \beta$ of $5,10,30,45$, and 55 . We find the remarkable result that one has now an upper limit on the chargino and sneutrino masses. Thus one finds that the sneutrino mass lies below $1.1 \mathrm{TeV}$ and the chargino mass lies below $590 \mathrm{GeV}$ for $\tan \beta=30$, the sneutrino mass lies below $1.4 \mathrm{TeV}$ and the chargino mass lies below $650 \mathrm{GeV}$ for $\tan \beta=45$, and the sneutrino mass lies below $1.5 \mathrm{TeV}$ and the chargino mass lies below $500 \mathrm{GeV}$ for $\tan \beta=55$. A similar situation occurs in the $m_{0}-m_{1 / 2}$ plane. In Fig. 2 we give a plot of the allowed region in the $m_{0}-m_{1 / 2}$ plane for $\tan \beta$ of $5,10,30,45$, and 55 . Here one finds an upper limit on $m_{0}$ of $1.1 \mathrm{TeV}$ and on $m_{1 / 2}$ of $750 \mathrm{GeV}$ for $\tan \beta=30$, an upper limit on $m_{0}$ of $1.4 \mathrm{TeV}$ and on $m_{1 / 2}$ of $800 \mathrm{GeV}$ for $\tan \beta=45$, and an upper limit on $m_{0}$ of $1.5 \mathrm{TeV}$ and on $m_{1 / 2}$ of $625 \mathrm{GeV}$ for $\tan \beta=55$. There are also interesting lower limits on the parameters in Figs. 1 and 2. Additionally, one finds that the allowed parameter space accommodates a light Higgs of $115 \mathrm{GeV}$ which is in the vicinity of the lower limit on the Higgs mass from the CERN Large Electron-Positron Collider data. We also note from Figs. 2(a) and 2(b) that $m_{0} \leq 550 \mathrm{GeV}$ and $m_{1 / 2} \leq 475 \mathrm{GeV}$, respectively, for $\tan \beta \leq 10$. The lower limits, e.g., of the light chargino mass, in this case are constrained only by experiment which for the light chargino is about $100 \mathrm{GeV}$. Thus in this part of the parameter space the light chargino could be accessible at the Fermilab collider Tevatron Run 2 (RUNII) since RUNII will be able to explore chargino masses up to about $200 \mathrm{GeV}$ via the trileptonic signal. Thus the implication is that if $\tan \beta \sim 5-10$, it would be much easier to discover sparticles at accelerators. However, there is no guarantee that we shall find ourselves in this region of the parameter space and thus the discovery of sparticles, though possible at the Tevatron, is not guaranteed. Returning to the upper limits we can say
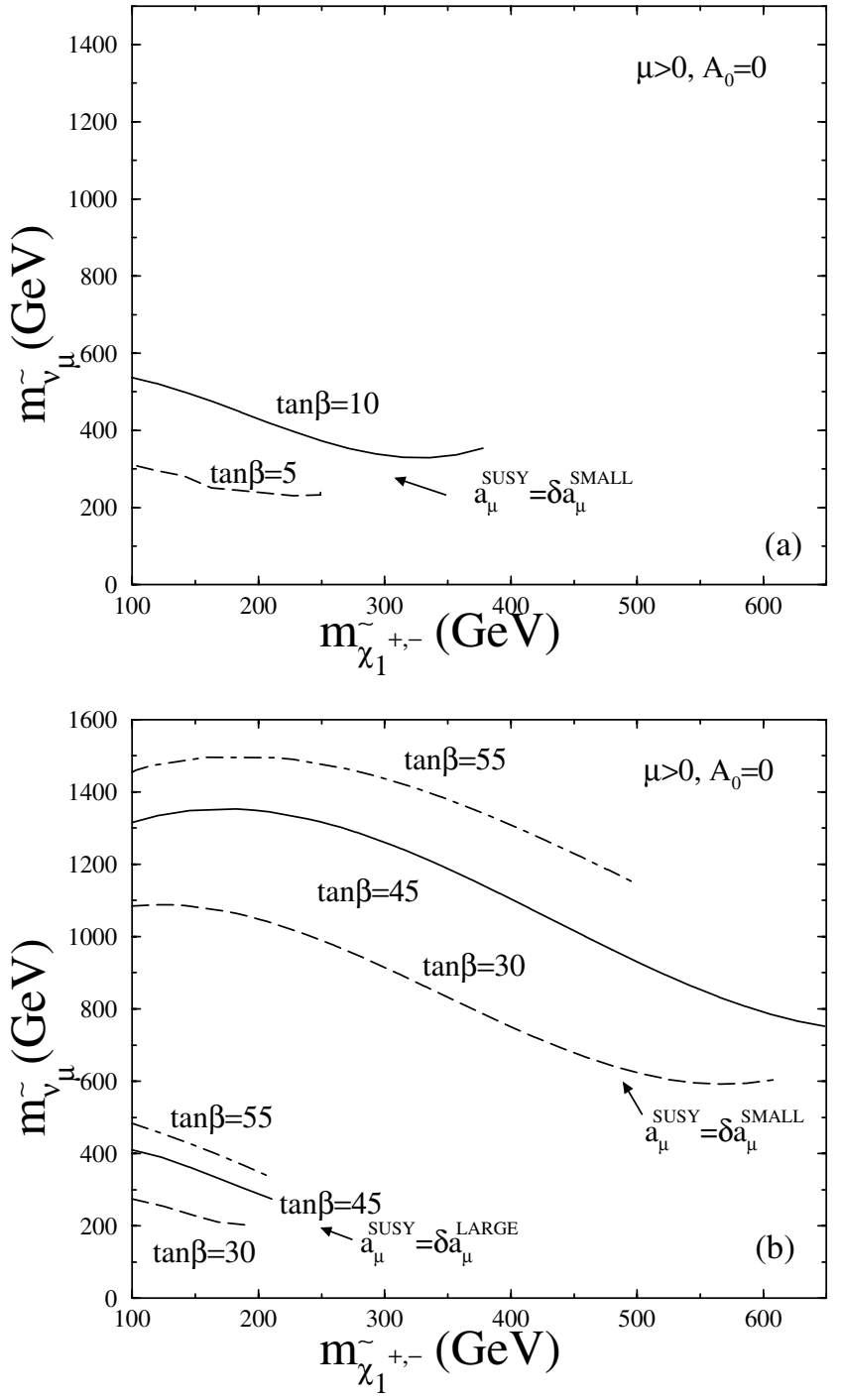

FIG. 1. A plot (a) of the upper limits in the sneutrino-light chargino plane for mSUGRA from the lower limit of $a_{\mu}^{\text {SUSY }}$ when $\tan \beta=5$ and $10, A_{0}=0$, and $\mu>0$. Similar plots (b) for $\tan \beta=30,45$, and 55 have both upper and lower limits. The $g-2$ bounds are $\delta a_{\mu}^{\mathrm{SMALL}}=10.6 \times 10^{-10}$ and $\delta a_{\mu}^{\mathrm{LARGE}}=$ $76.2 \times 10^{-10}$.

that under the constraint of the BNL $g-2$ experiment we find that

$$
m_{1 / 2} \leq 800 \mathrm{GeV}, \quad m_{0} \leq 1.5 \mathrm{TeV}(\tan \beta \leq 55) .
$$

Although more detailed mapping of the parameter space would modify the limits somewhat, we expect the main result to survive with some modest corrections. However, one must take the result of Eq. (3) with caution. We note that Eq. (1) exhibits a new physics effect at the $2.6 \sigma$ level and there were 4 times more data collected by the BNL group in the year 2000 which will be analyzed in the near future, and an important further check of the result will occur. To check if our upper limits of Eq. (3) are robust we have also analyzed the $g-2$ constraint within the minimal AMSB scenario following the analysis of Ref. [10] to which the reader is referred to for details and references. 

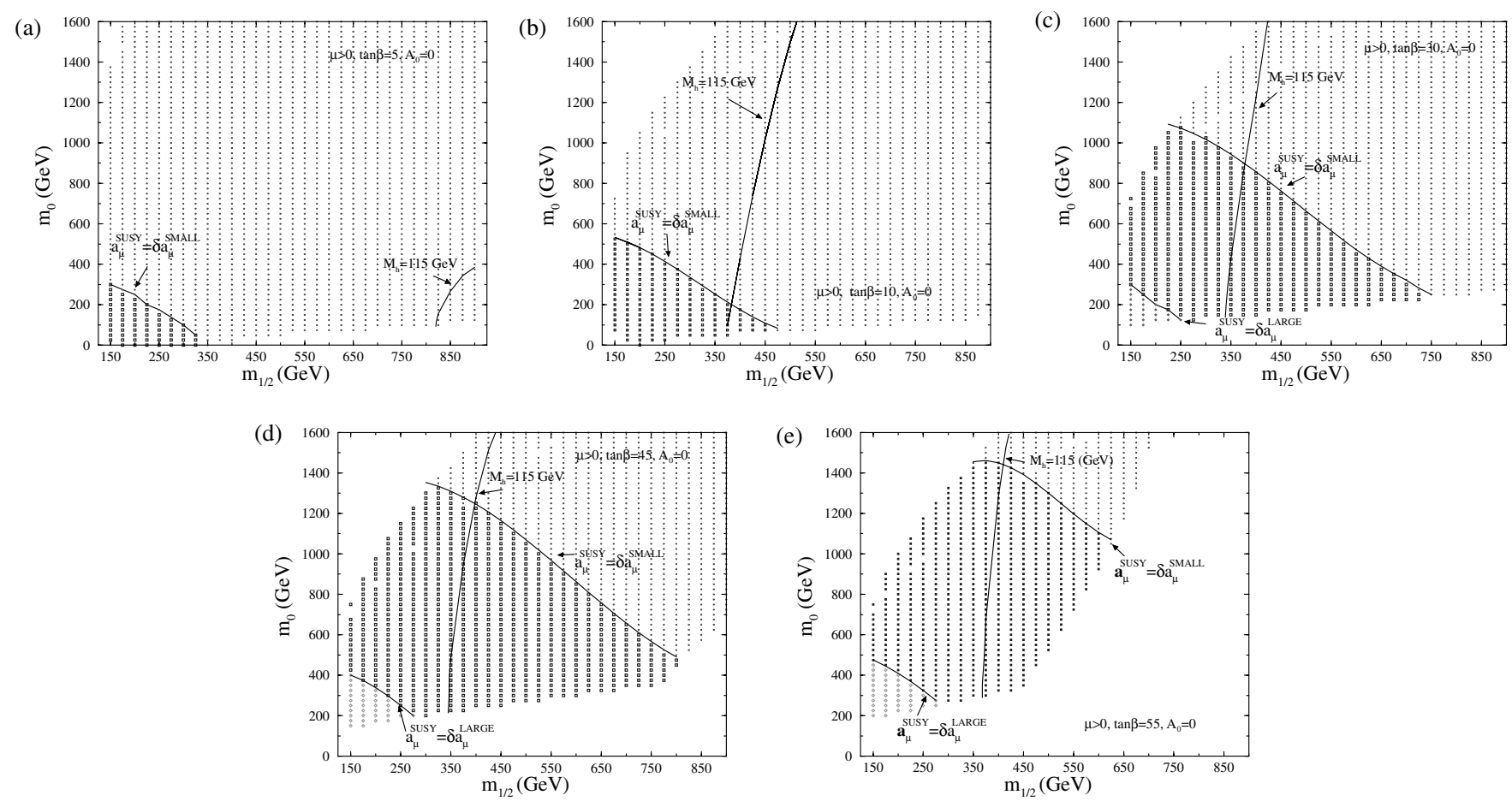

FIG. 2. Plots of the regions allowed (squares) by the $g-2$ constraint in the $m_{0}-m_{1 / 2}$ plane in mSUGRA when tan $\beta=5$ (a), 10 (b), 30 (c), 45 (d), and 55 (e), with $A_{0}=0$ and $\mu>0$.

The results are presented in Fig. 3 for $\mu>0$ and $\tan \beta=$ $10,30,40(\tan \beta=45$ in this case is not allowed because of the $C P$ odd Higgs boson turning tachyonic which in turn results from a large b-quark SUSY QCD correction [10]). Here one finds an upper limit on the chargino mass of about $300 \mathrm{GeV}$ and on the sneutrino mass of about $1.1 \mathrm{TeV}$. These limits are lower than those implied by Eq. (3). Thus the upper limits of Eq. (3) are very robust. Equation (3) implies an upper limit on the light chargino mass of about $650 \mathrm{GeV}$, and upper limits for the gluino mass and squark masses of about $2 \mathrm{TeV}$. Since the CERN Large Hadron
Collider (LHC) will be able to see the squarks and gluinos till about $2 \mathrm{TeV}$ [18], the above results provide strong evidence for the possibility that sparticles must become visible at the LHC. Further, an analysis of the effects of extra dimensions from Kaluza-Klein excitations on $g-2$ show that these do not provide a strong background to SUSY [19].

In conclusion, the BNL data provide a determination of the sign of the Higgs mixing parameter and we find this sign to be positive in the standard notation [15]. Further, assuming that the entire difference between experiment and
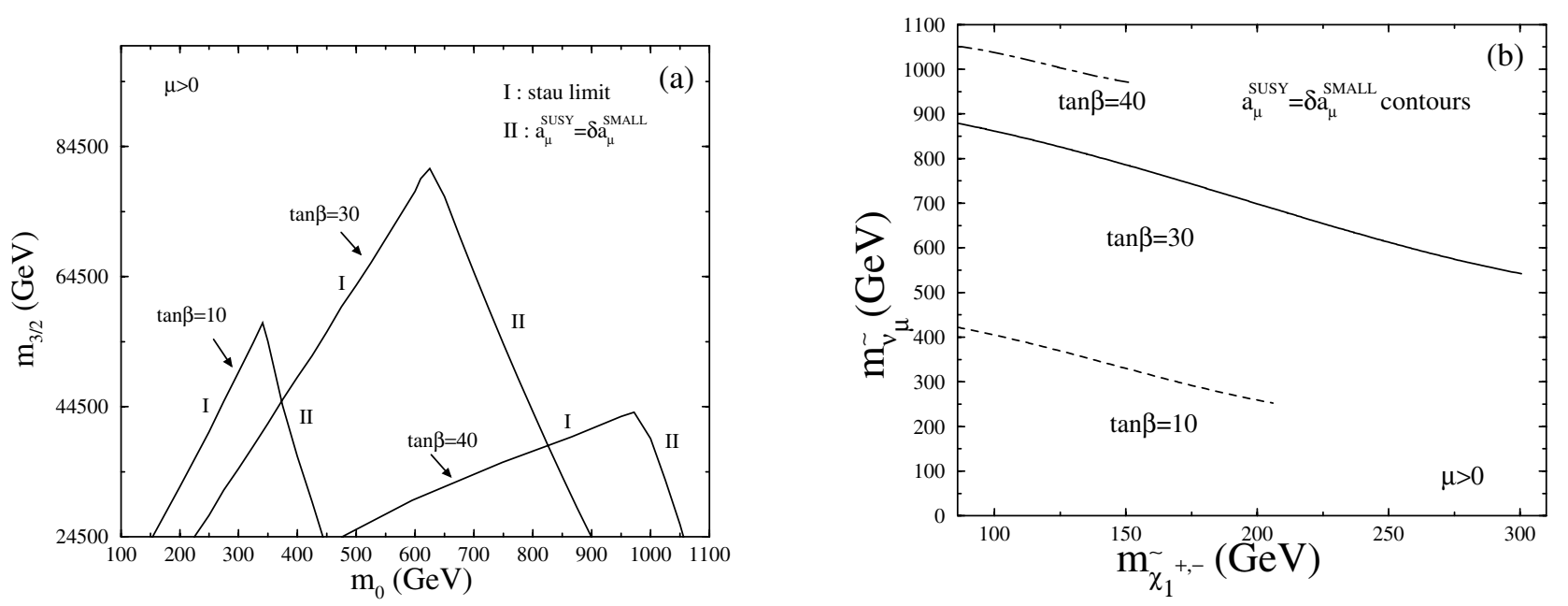

FIG. 3. (a) A plot of the region allowed (inside of the triangular areas) by the $g-2$ constraint in the $m_{3 / 2}-m_{0}$ plane for the minimal AMSB model when $\tan \beta=10,30$, and 40. The regions above the left sidearm of each triangle are disallowed due to stau turning tachyonic; (b) same allowed regions when plotted in the sneutrino-light chargino plane. 
the standard model result comes from SUSY, we find that the result from the Brookhaven experiment implies an upper bound on the SUSY parameters $m_{0}$ and $m_{1 / 2}$ which we find to lie in a region accessible to future accelerators. Thus we conclude that the major implication of the BNL $g-2$ result is that sparticles must become visible at the LHC and possibly at RUNII of the Tevatron. We also find that the parameter space allowed by the $g-2$ experimental limits allow a Higgs mass of $115 \mathrm{GeV}$ which is in the vicinity of the lower limit from LEP. The positive $\mu$ sign and the upper limits on the sparticles masses implied by the BNL $g-2$ data is also encouraging for the discovery of neutralino dark matter in dark matter searches. In the analysis above we did not impose the $b \rightarrow s+\gamma$ constraint and the relic density constraint. These are more model dependent and would tend to only reduce the upper limits of Eq. (3). Thus our upper limits of Eq. (3) and our prediction of the observation of sparticle at the LHC are robust.

This research was supported in part by NSF Grant No. PHY-9901057.

Note added.-After completion of this paper we noticed the following papers which appear to have some overlap with this work: Everett, Kane, Rigolin, and Wang [20]; Feng and Matchev [21]; Baltz and Gondolo [22].

*Permanent address: Department of Physics, Northeastern University, Boston, MA 02115.

[1] Muon $(g-2)$ Collaboration, H. N. Brown et al., hep-ex/ 0102017.

[2] A. Czarnecki and W. J. Marciano, Nucl. Phys. (Proc. Suppl.) B76, 245 (1999).

[3] M. Davier and A. Hocker, Phys. Lett. B 435, 427 (1998).

[4] P. Fayet, in Unification of the Fundamental Particle Interactions, edited by S. Ferrara, J. Ellis, and P. van Nieuwenhuizen (Plenum, New York, 1980), p. 587; J. A. Grifols and A. Mendez, Phys. Rev. D 26, 1809 (1982); J. Ellis, J. S. Hagelin, and D. V. Nanopoulos, Phys. Lett. 116B, 283 (1982); R. Barbieri and L. Maiani, Phys. Lett. 117B, 203 (1982).

[5] D. A. Kosower, L. M. Krauss, and N. Sakai, Phys. Lett. 133B, 305 (1983).

[6] T. C. Yuan, R. Arnowitt, A. H. Chamseddine, and P. Nath, Z. Phys. C 26, 407 (1984).
[7] K. Fujikawa, B. W. Lee, and A. I. Sanda, Phys. Rev. D 6, 2923 (1972); R. Jackiw and S. Weinberg, Phys. Rev. D 5, 2473 (1972); G. Altarelli, N. Cabbibo, and L. Maiani, Phys. Lett. 40B, 415 (1972); I. Bars and M. Yoshimura, Phys. Rev. D 6, 374 (1972); W. A. Bardeen, R. Gastmans, and B. E. Lautrup, Nucl. Phys. B46, 315 (1972).

[8] J. L. Lopez, D. V. Nanopoulos, and X. Wang, Phys. Rev. D 49, 366 (1994).

[9] U. Chattopadhyay and P. Nath, Phys. Rev. D 53, 1648 (1996); T. Moroi, Phys. Rev. D 53, 6565 (1996); M. Carena, M. Giudice, and C. E. M. Wagner, Phys. Lett. B 390, 234 (1997); K. T. Mahanthappa and S. Oh, Phys. Rev. D 62, 015012 (2000); T. Blazek, hep-ph/9912460.

[10] U. Chattopadhyay, D. K. Ghosh, and S. Roy, Phys. Rev. D 62, 115001 (2000).

[11] T. Ibrahim and P. Nath, Phys. Lett. B 418, 98 (1998); Phys. Rev. D 57, 478 (1998); T. Falk and K. Olive, Phys. Lett. B 439, 71 (1998); M. Brhlik, G. J. Good, and G. L. Kane, Phys. Rev. D 59, 115004 (1999); E. Accomando, R. Arnowitt, and B. Dutta, Phys. Rev. D 61, 075010 (2000).

[12] T. Ibrahim and P. Nath, Phys. Rev. D 61, 095008 (2000); 62, 015004 (2000).

[13] A. Czarnecki and W. J. Marciano, hep-ph/0102122.

[14] A. H. Chamseddine, R. Arnowitt, and P. Nath, Phys. Rev. Lett. 49, 970 (1982); R. Barbieri, S. Ferrara, and C. A. Savoy, Phys. Lett. 119B, 343 (1982); L. Hall, J. Lykken, and S. Weinberg, Phys. Rev. D 27, 235 (1983); P. Nath, R. Arnowitt, and A.H. Chamseddine, Nucl. Phys. B227, 121 (1983).

[15] SUGRA Working Group Collaboration, V. Barger et al., hep-ph/0003154.

[16] P. Nath and R. Arnowitt, Phys. Lett. B 336, 395 (1994); Phys. Rev. Lett. 74, 4592 (1995); F. Borzumati, M. Drees, and M. Nojiri, Phys. Rev. D 51, 341 (1995); H. Baer, M. Brhlik, D. Castano, and X. Tata, Phys. Rev. D 58, 015007 (1998).

[17] For a recent review, see R. Arnowitt, B. Dutta, and Y. Santoso, hep-ph/0102181.

[18] CMS Collaboration, Technical Proposal No. CERN/LHCC 94-38, 1994; ATLAS Collaboration, Technical Proposal No. CERN/LHCC 94-43, 1944; H. Baer, C-H. Chen, F. Paige, and X. Tata, Phys. Rev. D 52, 2746 (1995); Phys. Rev. D 53, 6241 (1996).

[19] P. Nath and M. Yamaguchi, Phys. Rev. D 60, 116006 (1999).

[20] L. L. Everett, G. L. Kane, S. Rigolin, and L-T. Wang, Phys. Rev. Lett. 86, 3484 (2001).

[21] J. L. Feng and K. T. Matchev, Phys. Rev. Lett. 86, 3480 (2001).

[22] E. A. Baltz and P. Gondolo, Phys. Rev. Lett. 86, 5004 (2001). 